\title{
Viscosity Determination in the Earth's Outer Core from ScS and SKS Phases
}

\author{
By \\ Yasunori SuzukI and Ryosuke SATO \\ Geophysical Institute, Faculty of Science, University of Tokyo
}

\begin{abstract}
Attenuation of seismic waves in the Earth's outer core is investigated by using pairs of transmitted waves through the outer core and reflected waves at the coremantle boundary, which have common ray paths in the mantle.

Taking into account the conditions: that the angle of incidence at the core-mantle boundary is small so that ray theory is applicable; that the phase to be analyzed should be clear and not contaminated by other phases; and that the ray does not penetrate into the inner core; pairs of $S K S$ and $S c S$ are used to determine the viscosity coefficient in the outer part of the Earth's outer core.

Long-period seismograms of three deep-focus earthquakes supplied by the U.S.C. G.S. are used in this analysis. Ratios of the Fourier spectral amplitudes of SKS to those of $S c S$ in the frequency range of $0.04 \mathrm{~Hz}$ to $0.15 \mathrm{~Hz}$, after eliminating the effects of crustal layering and the seismograph response, yield a mean viscosity coefficient $\bar{\nu}$ in the outer core. Using 16 pairs of $S K S$ and $S C S$ waves $\bar{\nu}$ in the above frequency range is estimated to be $3 \times 10^{10}$ to $7 \times 10^{10}$ dyne $\cdot \mathrm{sec} / \mathrm{cm}^{2}$, which is consistent with the $\bar{\nu}$ determination from multiply-reflected ScS's at the core-mantle boundary.
\end{abstract}

\section{§1. Introduction}

Recently, studies on attenuation of seismic waves in the mantle have been made by various investigators, for example, ANDERSON and ARCHAMBEAU (1964), ANDERSON and KOVACH (1964), KOVACH and ANDERSON (1964), ANDERSON et al. (1965), TENG (1966, 1968), KURITA (1966), HIRASAWA and TAKANO (1966), SATO and EspinOSA (1967), MIKUMO and KURITA (1968), using long-period seismic wave recordings; and TAKANO (1966), KANAMORI (1967 a, b, c), SUMNER (1967), and KuRITA (1968), using short-period seismic wave recordings. However, the detailed $Q$-structure in the mantle has not been established as yet, and further work is required to ascertain the variations of $Q$ with depth.

There have been several proposed speculations in the constitution of the physical properties of the core, but there are quite a number of unknown parameters. So far, one of the few accepted properties is that the rigidity of the outer core is negligible, if it exists, and in this sense the outer core is regarded to be liquid.

The velocity distribution in the core has been studied by several investigators, such as JEFFREYS (1939), GUTENBERG (1959), BOLT (1964), ADAMS and RANDALL (1964), and their overall results are similar structures, at least in the outer core. For the inner core, however, it may be stated that the physical properties, even velocity distribution, are still unknown. Taking into account the actual knowledge of the inner and outer core, it was decided in the present study to investigate the attenuation of seismic waves traveling through the outer core only, eliminating the effects of attenuation in the mantle.

There have been several attempts to obtain the rigidity of the core assuming that it is a soft-solid, or the viscosity of the core assuming that it is a viscous-liquid medium. One of these estimations has been done by determining a change of period of the free oscillation of the earth, and some other studies from the reflectivity of seismic body waves 
at the core-mantle boundary. These studies are summarized in the paper by SATO and ESPINOSA (1967). There have been no investigations to directly determine the attenuation of seismic waves by analyzing records of waves transmitted through the Earth's outer core.

In this study the outer core is considered to be a viscous-liquid medium, and the mean viscosity is determined from the spectral amplitude ratios of waves transmitted through the outer core and waves reflected at the core-mantle boundary, which have common ray paths in the mantle. An important question concerned with the physical properties of the core arises whether the attenuation in the outer core behaves like that in a solid; where the attenuation factor is proportional to frequency (the dimensionless quality factor $Q$ is independent of frequency), or it behaves like that in a viscous-liquid medium; where the attenuation factor is proportional to the square of the frequency (the viscosity coefficient $\nu$ is independent of frequency).

The applicability of a constant $Q$-model for the outer core in the frequency rang under consideration is also discussed.

\section{§2: Method of Analysis}

In Fig. 1 is shown schematically a transmitted wave through the outer core and a reflected wave at the core-mantle boundary which have common ray paths in the mantle. The take-off angle at the focus, the angle of incidence at the core-mantle boundary, $\theta_{C}$, and at the Mohorovicic discontinuity, $\theta_{M}$, as used in this study are shown in this figure.

The observed amplitude spectra of the outer core phase, $A_{C}(f)$, and of the core-reflected phase, $A_{R}(f)$, are expressed by

$A_{C}(f)=A_{0}(f) \cdot P_{C} \cdot T \cdot D_{C} \cdot C_{C}(f) \cdot S_{C}(f) \cdot X_{C}(f)$

and

$A_{R}(f)=A_{0}(f) \cdot P_{R} \cdot R \cdot D_{R} \cdot C_{R}(f) \cdot S_{R}(f) \cdot X_{R}(f)$

respectively, where

$\begin{array}{ll}f: & \text { frequency, } \\ A_{0}(f): & \text { source spectrum, } \\ P_{C} \text { and } P_{R}: & \text { radiation pattern effects, } \\ T \text { and } R: & \begin{array}{l}\text { transmission and reflection } \\ \text { coefficients, at the core-mantle } \\ \text { boundary, }\end{array} \\ D_{C} \text { and } D_{R}: \quad \begin{array}{l}\text { divergence factors due to geo- } \\ \text { metrical spreading, }\end{array} \\ C(f) \quad: \begin{array}{l}\text { transfer function of the crustal } \\ \text { layering, }\end{array} \\ S(f) \quad: \begin{array}{l}\text { amplitude response of seismo- } \\ \text { graph system, }\end{array} \\ \text { effect of attenuation which is } \\ \text { given below. }\end{array}$

If the attenuation is like that in a solid and $Q$ is frequency independent, then

$$
X(f)=\exp \left(-\pi f \int_{\text {ray path }} \frac{d s}{Q V}\right)
$$

and if the attenuation is like that in a viscousliquid medium and the viscosity coefficient $\nu$ is frequency independent, then

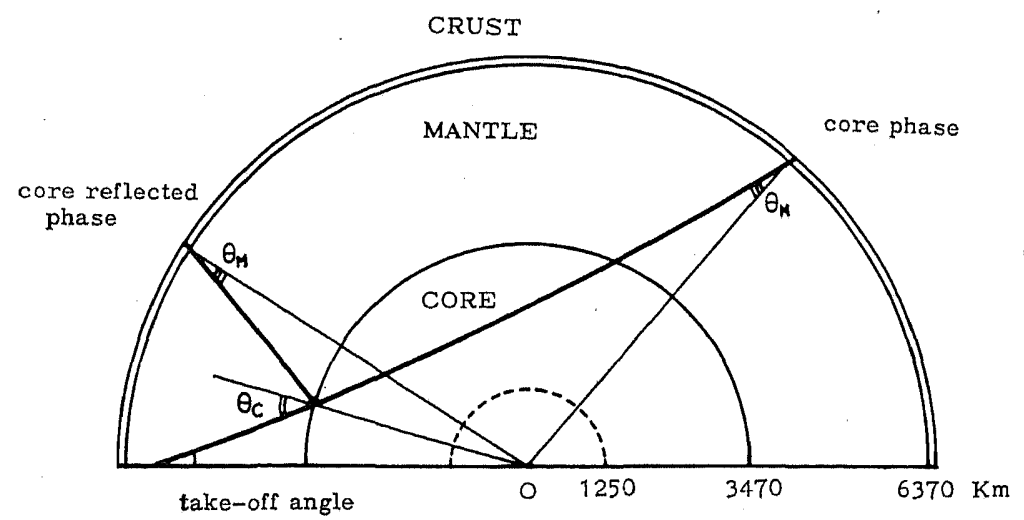

Fig. 1. A pair of core phase and core reflected phase (A schematic picture). 


$$
X(f)=\exp \left(-2 \pi^{2} f^{2} \int_{\text {ray path }} \frac{\nu d s}{\rho V^{3}}\right)
$$

where $V$ is the seismic wave velocity, $\rho$ is the density, $d s$ is the ray path element and the integral is performed along the entire ray path.

Now let us define the modified ratio $A$ of the amplitude spectra by

$$
A \equiv \frac{A_{C}(f) / C_{C}(f) \cdot S_{C}(f)}{A_{R}(f) / C_{R}(f) \cdot S_{R}(f)}
$$

From equation (1) and (2), we obtain

$$
A=\left(\frac{P_{C} \cdot T \cdot D_{C}}{P_{R} \cdot R \cdot D_{R}}\right) \frac{X_{C}(f)}{X_{R}(f)}
$$

We may reasonably regard the factor in parenthesis in the right-hand side of equation (4) as frequency independent, so far as we use the phases for which ray theory is applicable. The factor $X_{C}(f) / X_{R}(f)$ is reduced to the attenuation effect in the outer core only, because the phases forming the pair have almost the same paths in the mantle and so the attenuation effects in the mantle are naturally the same. We will discuss the effects of lateral inhomogeneity in the upper mantle in section 5 .

If the outer core is a viscous-liquid medium, equation (4) becomes"

$$
A=\text { const } \cdot \exp \left[-2 \pi^{2} f^{2} \bar{\nu} \int_{\substack{\text { ray path in } \\ \text { the outer corc }}} \frac{d s}{\rho V^{3}}\right]
$$

where $\bar{\nu}$ is mean viscosity coefficient in the outer core.

Then,

$$
\begin{aligned}
\ln A & =\text { const }-2 \pi^{2} f^{2} \bar{\nu} \int \frac{d s}{\rho V^{8}} \\
& =\text { const }-f^{2} \bar{\nu} J
\end{aligned}
$$

where

$$
J=2 \pi^{2} \int_{\substack{\text { rayy path in } \\ \text { the outer core }}} \frac{d s}{\rho V^{3}}
$$

$J$ as defined by equation (7) can be calculated from a given density and velocity structure in the outer core. Taking the Jeffreys-Bullen structure, $J$ is shown in Fig. 5 as a function of angle of incidence at the core-mantle boundary. The use of different structures in the outer core yields only slight differences in $J$.

According to the method described above, both pairs of $P K P-P c P$ and $S K S-S c S$ seem to be adequate for the study of the attenuation in the outer core. But the pair of PKP-PcP is inadequate by the following reasons:

1. In order that we can take the transmission and reflection coefficients at the coremantle boundary as frequency independent, the angle of incidence $\theta_{C}$ should be small. SATo (1969) showed that ray theory is not applicable even for waves of period less than $20 \sim 30 \mathrm{sec}$, if $\theta_{C}$ is greater than $70^{\circ}$, and

2 . the $P K P$ phase should not be contaminated by $P K I K P$ propagated through the inner core.

The epicentral distance ( 4 ) for PKP phases which satisfy the above two conditions is limited to a narrow epicentral distance range at about $150^{\circ}$.

On the other hand for the pair of $S K S-S c S$ waves, the appropriate range of $\Delta$ for $S K S$ phases is from $70^{\circ}$ to $120^{\circ}$, and that for $S c S$ phases is from $15^{\circ}$ to $55^{\circ}$. Except at about $80^{\circ}$ where $S K S, S$ and $S K K S$ arrive at the station with very small time intervals, and at about $40^{\circ}$ where $S c S$ and $S S$ arrive almost

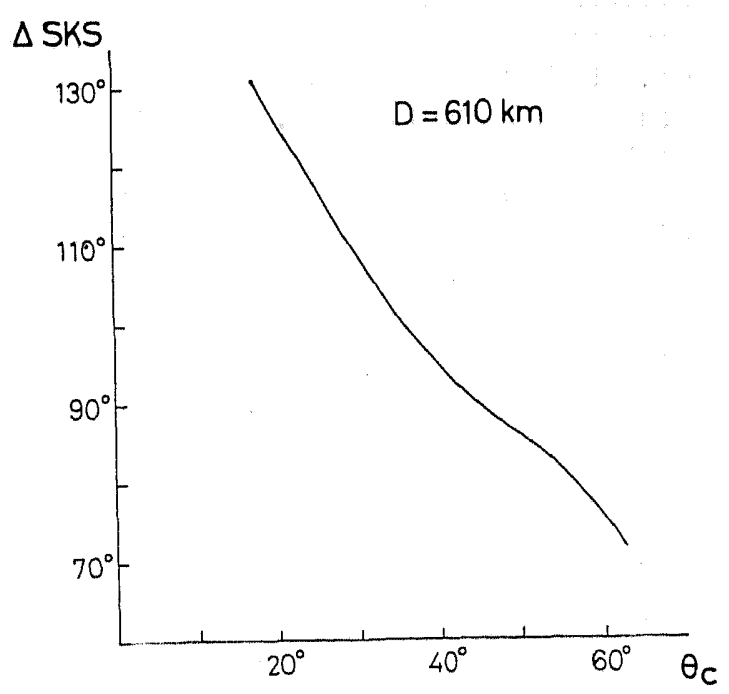

Fig. 2. An example of nomogram to find a relation $\Delta_{S K S}$ and $\theta_{C}$ for a focal depth of $610 \mathrm{~km}$ 


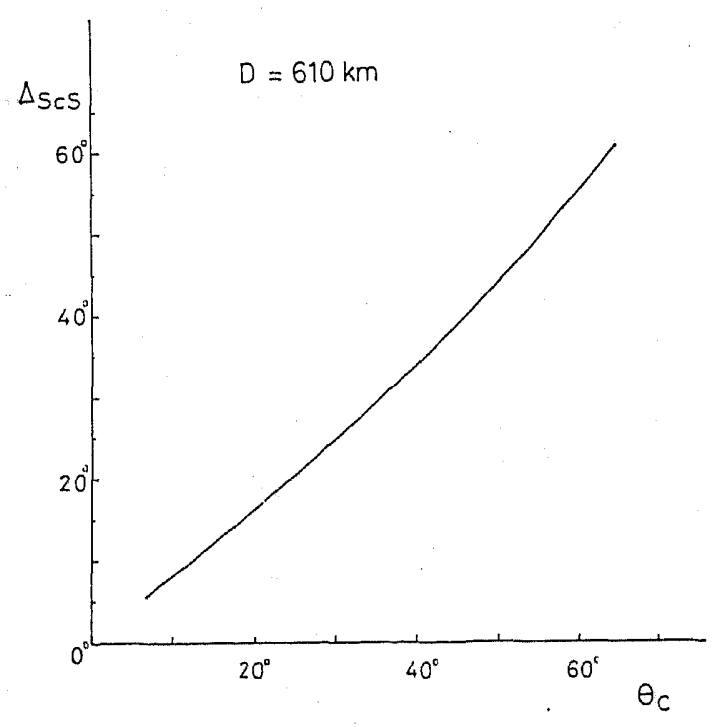

Fig. 3. $\Delta_{S o S}$ versus $\theta_{C}$.

simultaneously. As is shown in Figs. 2 and 3, $\theta_{C}$ for $S K S$ and $S c S$ in the above epicentral range is from $20^{\circ}$ to $45^{\circ}$ and so ray theory is applicable for these phases. Taking into account that even if $\Delta$ for SKS changes by several degrees, the corresponding change of the angle at the hypocenter is only 1 to 2 degrees, hence we can make use of several observations of SKS for one observation of $S c S$. In Fig. 4 are shown $\Delta_{S K S}$ and $\Delta_{S o S}$ as a function of the take-off angle for a focal depth of $610 \mathrm{~km}$. When the $S K S$ and $S c S$ have the same take-off angle they make a proper pair.

According to the reasons and constraints mentioned above, pairs of $S K S$ and $S c S$ are analyzed in this study.

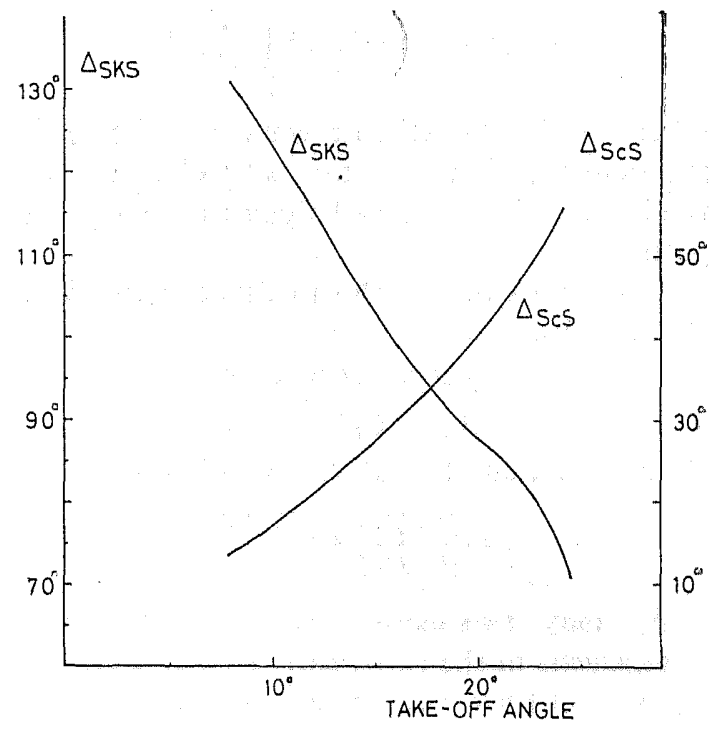

Fig. 4. An example of nomogram to obtain an appropriate pair of $S K S$ and $S c S$ which have the same take-off angle at the source.

\section{§3. Data}

In Table $I$ are listed three earthquakes used in the present study. The origin times, epicenters, focal depths, body-wave magnitudes $m_{b}$ are taken from the Seismological Bulletin of the U.S.C.G.S. Several nomograms are prepared for these earthquakes to obtain the appropriate pairs of $S K S$ and $S c S^{n}$ and to find the angles of incidence at Moho and at the core boundary for the phases, such as those shown in Figs. 2 through 5.

Since it is required that both $S K S$ and $S c S^{n}$ phases should be clearly recorded, the earthquakes selected are deep focus $(590 \sim 640 \mathrm{~km})$ and are fairly large magnitudes $\left(m_{b}=6.2\right)$. The analyzed records are from long-period

Table 1. Information of the earthquakes analysed (USCGS)

\begin{tabular}{c|l|l|c|c}
\hline $\begin{array}{c}\text { Shock } \\
\text { No. }\end{array}$ & Origin Time & Location & Depth (Km) & $m_{b}$ \\
\hline 1 & $\begin{array}{l}\text { Dec. } 28,1964 \\
16 \mathrm{~h} 16 \mathrm{~m} 11.0 \mathrm{~s}\end{array}$ & $\begin{array}{l}22^{\circ} .1 \mathrm{~S}, 179^{\circ} .6 \mathrm{~W} \\
\text { South of Fiji Islands }\end{array}$ & 611 & 6.2 \\
\hline 2 & $\begin{array}{l}\text { Mar. } 17,1966 \\
15 \mathrm{~h} 50 \mathrm{~m} 33.1 \mathrm{~s}\end{array}$ & $\begin{array}{l}21^{\circ} .1 \mathrm{~S}, 179^{\circ} .2 \mathrm{~W} \\
\text { Fiji Islands Region }\end{array}$ & 639 & 6.2 \\
\hline 3 & $\begin{array}{l}\text { Nov. } 3,1965 \\
01 \mathrm{~h} 39^{\mathrm{m}} 0.31 \mathrm{~s}\end{array}$ & $\begin{array}{l}9^{\circ} .1 \mathrm{~S}, 71^{\circ} .4 \mathrm{~W} \\
\text { Peru-Brazil Border Region }\end{array}$ & 593 & 6.2 \\
\hline
\end{tabular}




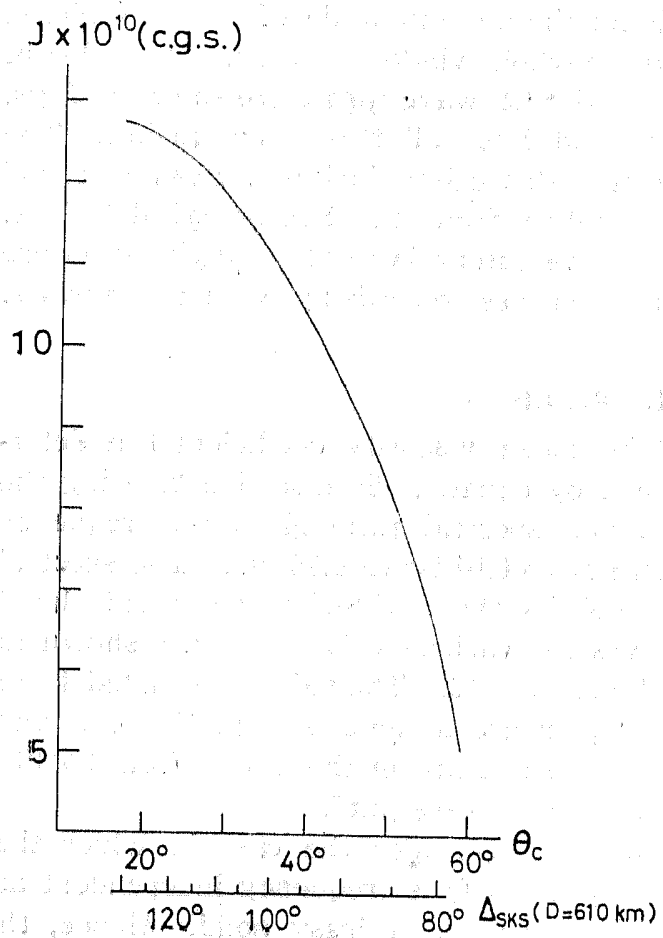

Fig. ${ }_{2} 5$. $J$, defined by equation (7), versus $\theta_{C}$. The lowermost scale indicatas $\Delta_{S K S}$ for a focal depth of $610 \mathrm{~km}$. horizontal component seismograms obtained at WWSSN stations. Since the $S V$ com. ponents are more adequate than the $S H$ components for our study, we tried to analyze the longitudinal components rather than the transverse components. When it was impossible to utilize the $S V$ components, we were forced to use the SH components. There were only two such cases out of twenty-four.

The epicentral distances and azimuths seen from the epicenter are shown for earthquake No. 1 in Fig. 6. The stations are indicated by the three letter codes, of the U.S.C.G.S. The records at the stations in brackets are analyzed, but were not used for the determination of the mean viscosity $\bar{\nu}$.

Since the free periods of the pendulum and galvanometer are reported as the same for all the stations, $30 \mathrm{sec}-100 \mathrm{sec}$ at the time of No. 1 earthquake and $15 \mathrm{sec}-100 \mathrm{sec}$ at the time of No. 2 and No. 3 earthquakes, the seismograph responses were assumed to be the same. So the factor $S_{C}(f) / S_{R}(f)$ in equation (3) was set equal to 1 .

Crustal structures are determined as follows. The $P$ wave velocity and layer thick-

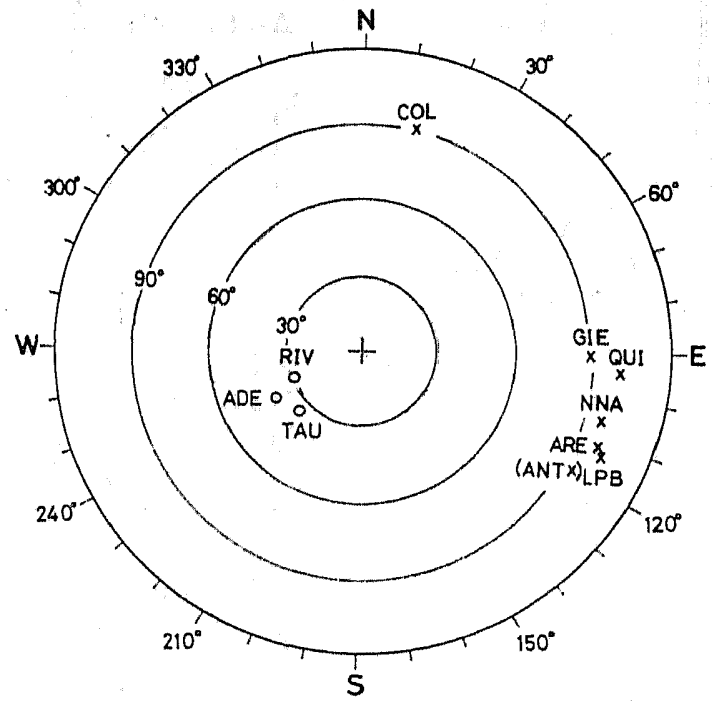

$$
\text { NO.1 } \quad \text { O SCS }
$$

Fig. 6. Azimuth and epicentral distance of stations seen from the epicenter (No. 1 earthquake). 
ness are taken from a compiled result by StEINHART and MEYER (1961), and the density is obtained by using NAFE and DRAKE's velocity-density curve (TALWANI et al., 1959). The appropriate Poisson's ratio was used to calculate $S$ wave velocity.

Travel times of $S K S$ and $S c S$ phases for each earthquake are all in good agreement with those of the Seismological Tables of JEFFREYS and BULLEN (1967).

The original films are enlarged by a factor of 40 , and digitized at every $1 / 4$ or $1 / 2$ seconds. The following data window is applied with $T_{m}=25 \sim 30$ seconds ;

$$
\begin{array}{rlrl}
w(t) & =\frac{1}{2}\left(1+\cos \frac{\pi t}{T_{m}}\right) ; & |t|<T_{m} \\
& =0 \quad ;|t|<T_{m}
\end{array}
$$

Except for one case, the onsets of the phases are taken as the origin time of analysis, which implies the signals are assumed to be zero before the onsets. The onset is not sharp in the case of the $S c S$ at RIV of earthquake No. 2, so the origin is set about the maximum amplitude of the $S c S$ phase, and the whole window is used. Examples of $S c S$ and $S K S$ wave-forms are shown in Figs. 7,8 , and 9 for all three earthquakes. The arrow with the J-B mark indicates the arrival time taken from the Seismological Tables. The time intervals used for digitization are: shown on these records by two small arrows.

\section{§4. Results}

The mean viscosity coefficient $\bar{\nu}$ is determined by equation (6) in section 2 , using the modified spectral ratio $A$ in the frequency range from $0.04 \mathrm{~Hz}$ to $0.15 \mathrm{~Hz}$. The spectral ratio $A$ for the $S K S-S c S$ pair and calculated curves for various values of $\bar{\nu}$ are shown in. in Figs. 10 to 12 . The values estimated from. each pair are summarized in Table 2 and most of them are in the range from $3 \times 10^{10}$. to $7 \times 10^{10}$ dyne $\cdot \mathrm{sec} / \mathrm{cm}^{2}$.

Next we consider the case in which the quality factor $Q$ is frequency independent as in the case for a lossy solid. Hence, the

NO. 1

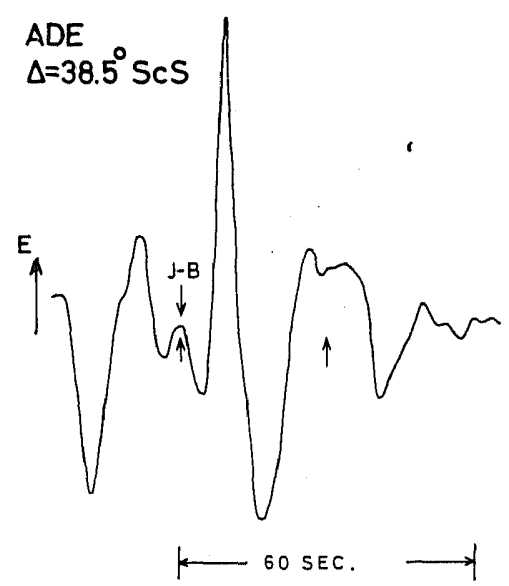

COL

$\Delta=90.1^{\circ}$ SKS

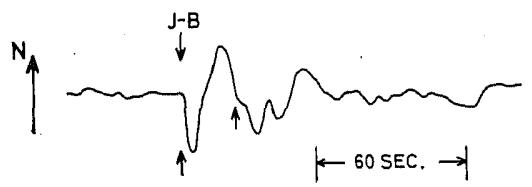

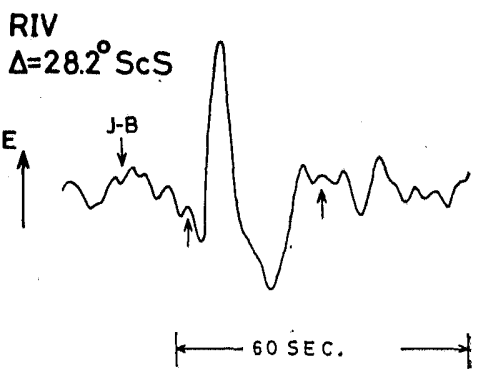

ARE

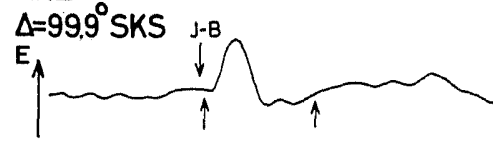

QUI

$\Delta=100.2^{\circ} \mathrm{SKS}$

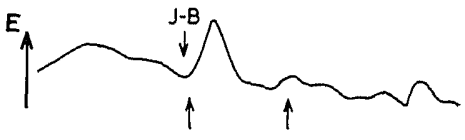

Fig. 7. Waveforms of $S c S$ and $S K S$ from the Dec. 28, 1964 earthquake (No. 1). 
NO. 2

ADE

$\Delta=39.3^{\circ} \mathrm{ScS}$

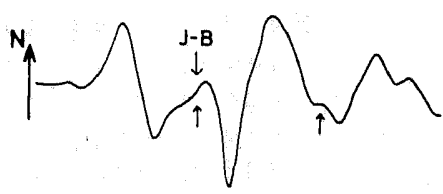

ALQ

$\Delta=88.7^{\circ} \mathrm{SKS}$

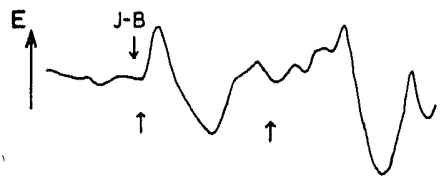

$\longleftarrow \quad 60$ SEC.
RIV

$\Delta=29.0^{\circ} \mathrm{ScS}$

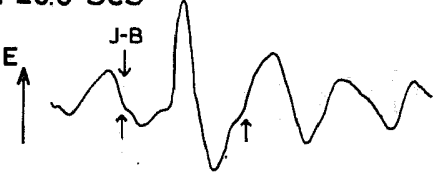

ARE $\Delta=99.9^{\circ}$ SKS
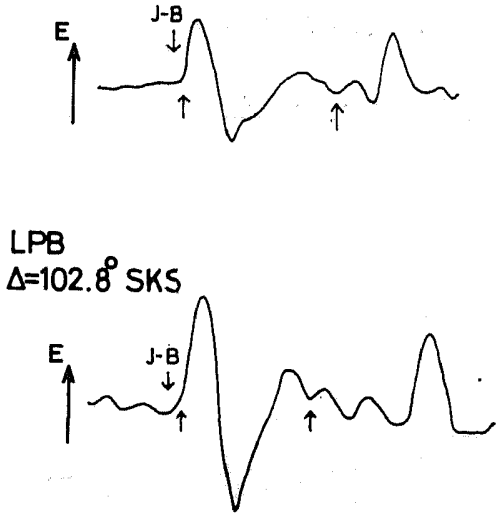

Fig. 8. Waveforms of $S C S$ and SKS from the Mar. 17, 1966 earthquake (No. 2).

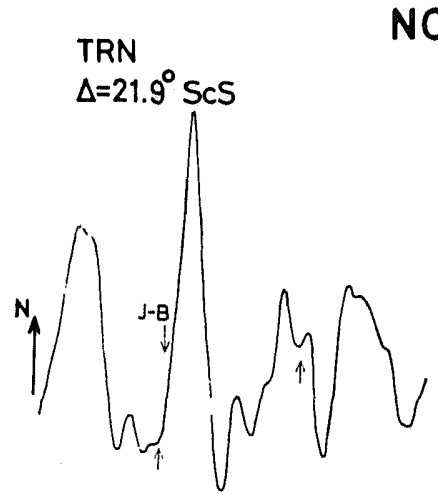

NAI

$\Delta=109.7^{\circ}$ SKS

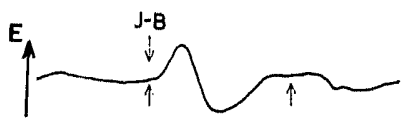

K $60 \mathrm{sEC}$.
NO. 3

JCT $\Delta=47.9^{\circ} \mathrm{ScS}$
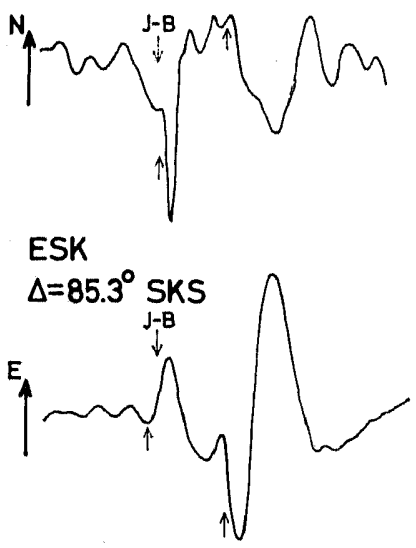

Fig. 9. Waveforms of $S c S$ and $S K S$ phases from the Peru-Brazil earthquake of Nov. 3. 1965 (No. 3). 


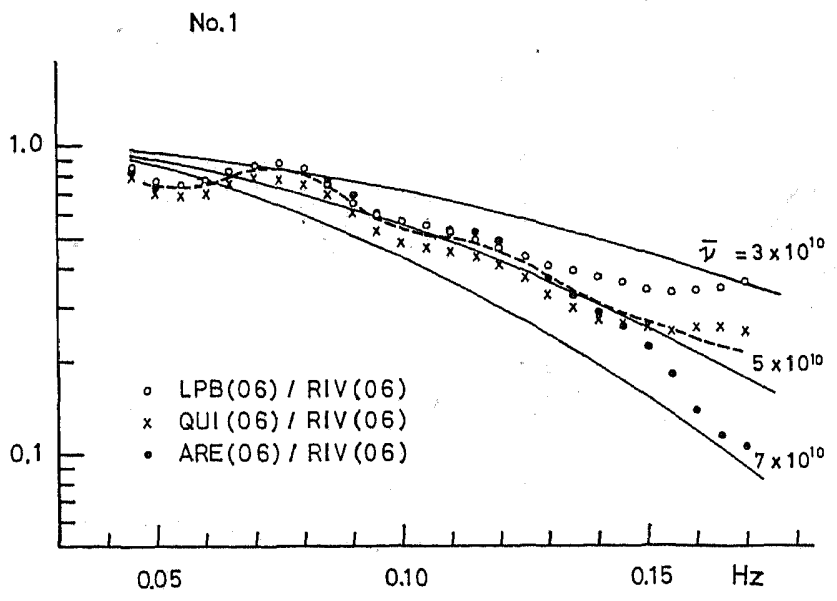

Fig. 10. a) Determination of $\bar{\nu}$ assuming that it is frequency independent in the frequency range under consideration. Solid curves indicate theoretical curves for a given $\bar{\nu}$ value, and a broken curve is the mean of the modified spectral ratios of three pairs (earthquake No. 1).

No.1

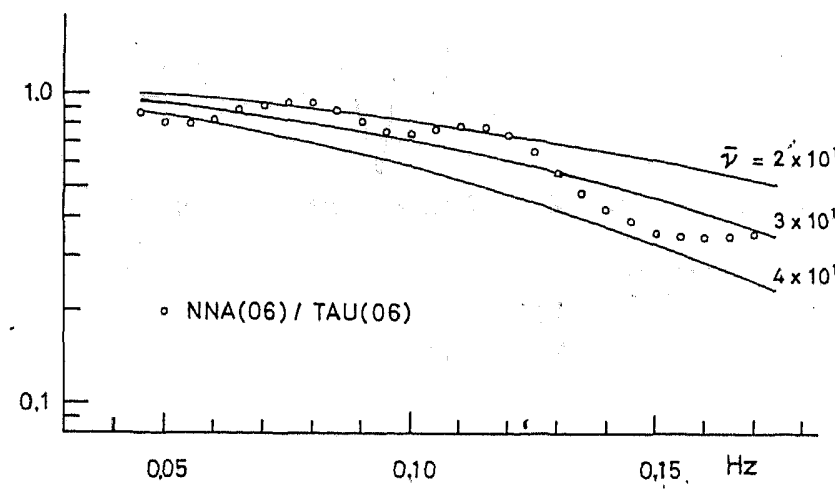

Fig. 10. b) Modified spectral ratio of $S K S$ and $S c S$ for earthquake No. 1 and theoretical curves for constant $\bar{\nu}$.

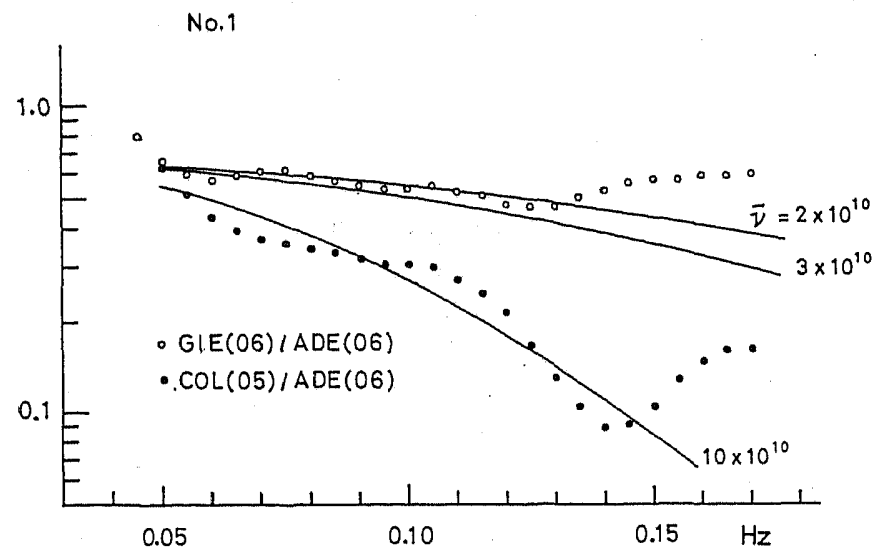

Fig. 10. c) $\bar{\nu}$ determination from earthquake No. 1. A somewhat large difference between two $\bar{\nu}$ values obtained from two pairs may be due to large difference in azimuthal direction of COL from other stations (cf. Fig. 6). 
No. 2

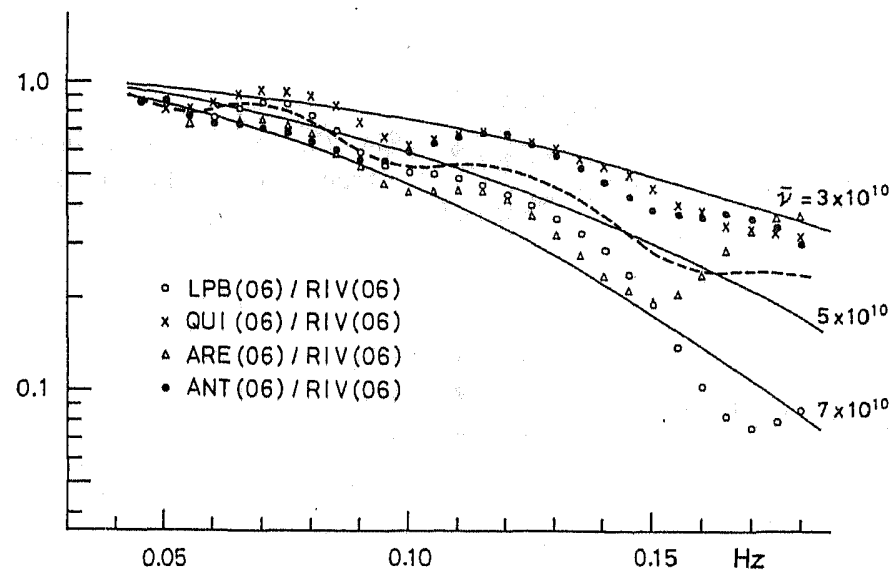

No.2

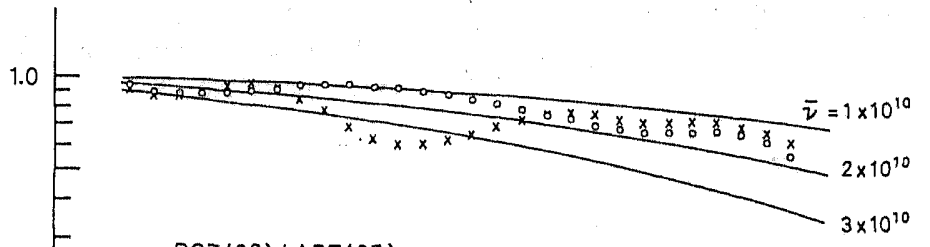

- $B O Z(06) / A D E(05)$

$\times A L Q(06) / A D E(05)$

0.1
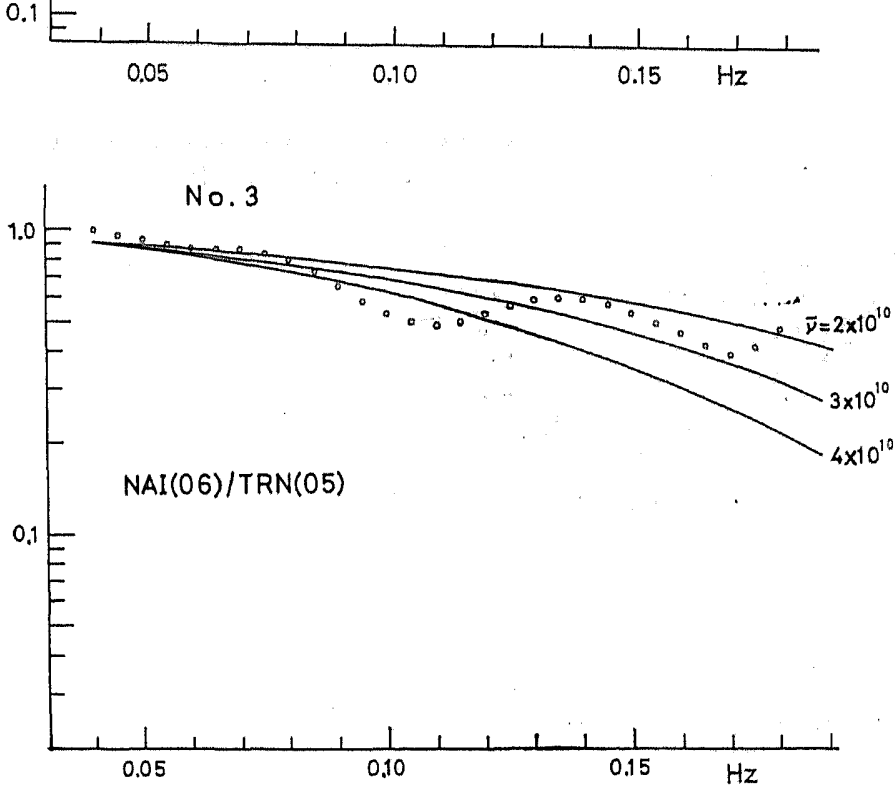

Fig. 11. a) $\nabla$ determination from earthquake No. 2.

Fig. 11. b) D determination from earthquake No. 2.

Fig. 12. a) $\bar{\nu}$ determination from earthquake No. 3 . 


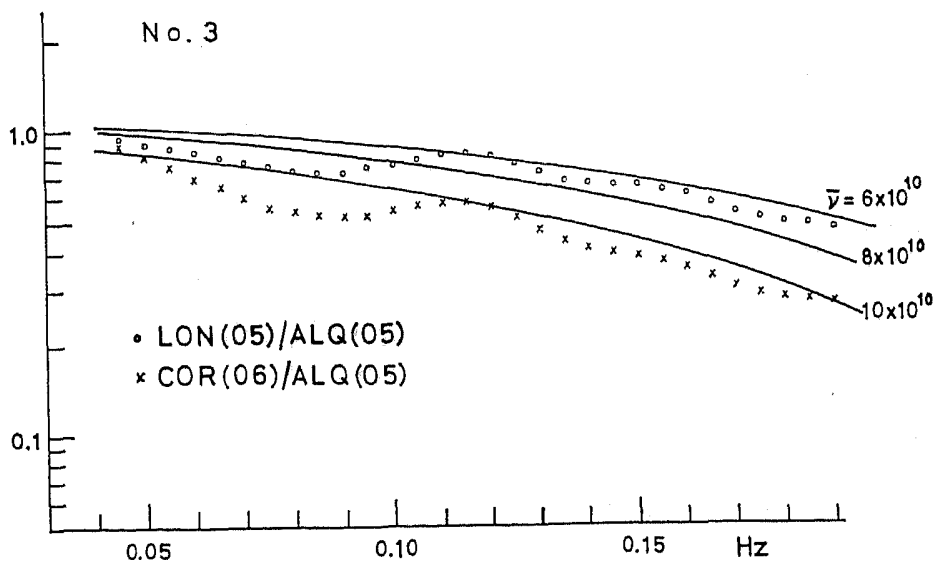

Fig. 12. b) $\bar{\nu}$ determination from earthquake No. 3 .

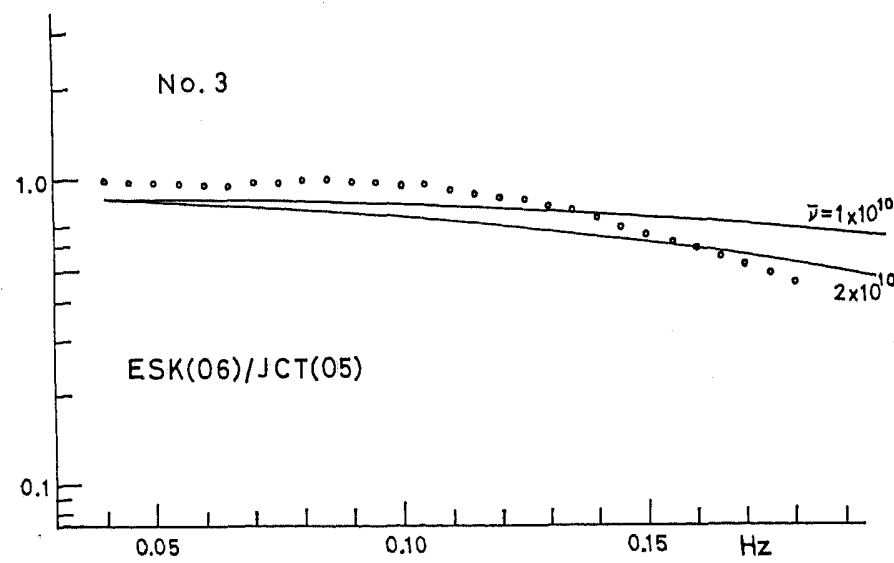

Fig. 12. c) $\bar{\nu}$ determination from earthquake No. 3.

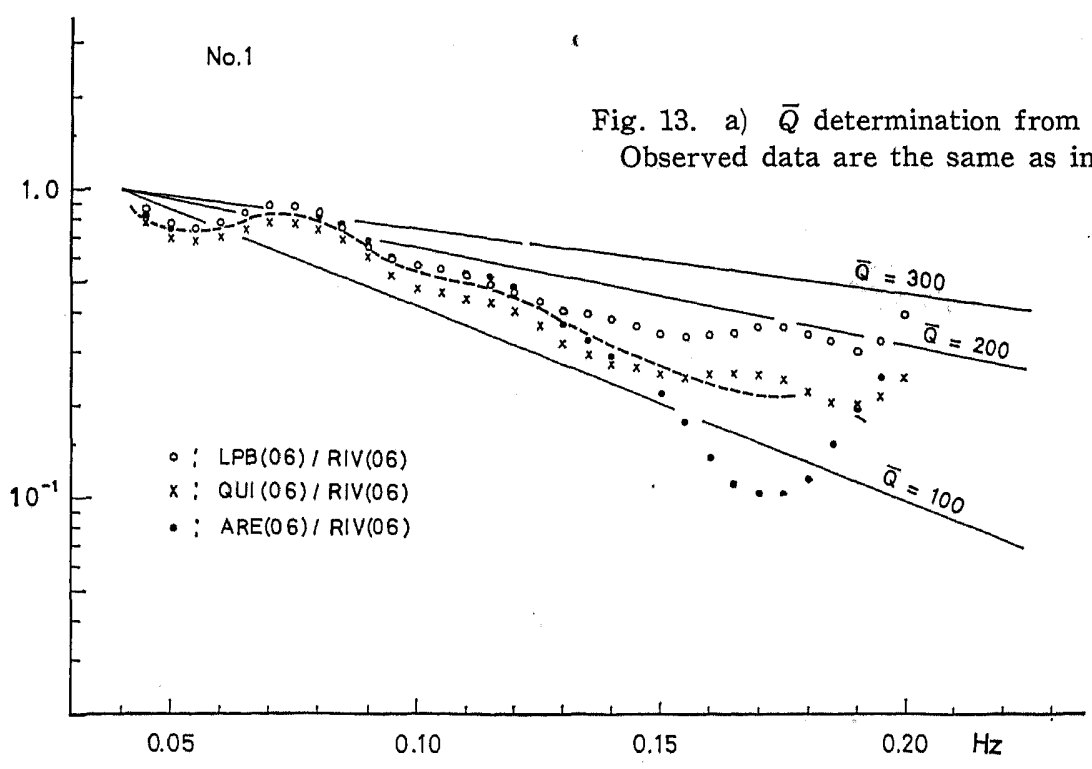




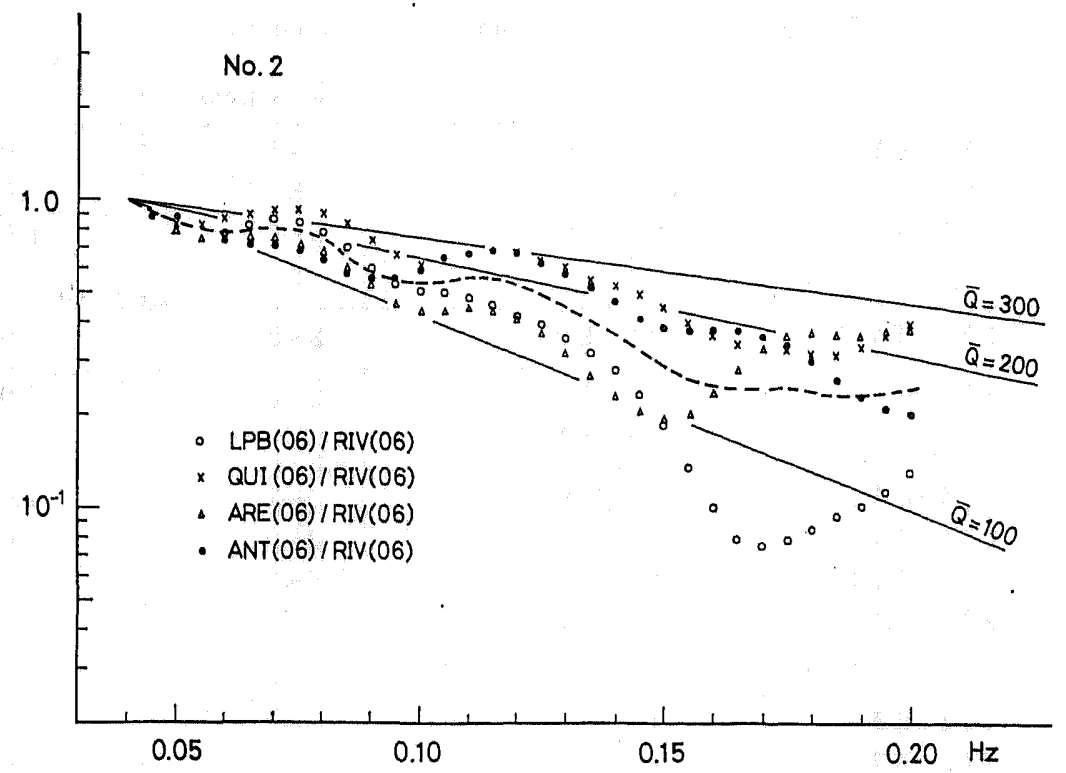

Fig. 13. b) $\bar{Q}$ determination from earthquake No. 2. Observed data are the same as in Fig. 11. a).

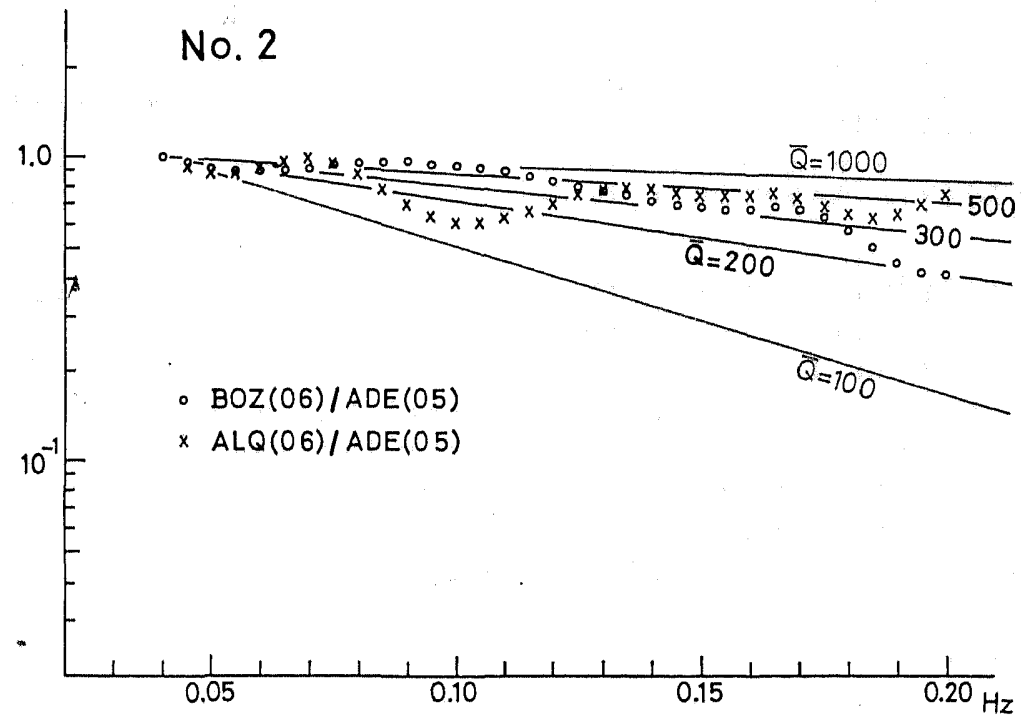

Fig. 13. c) $\bar{Q}$ determination from earthquake No. 2. Observed data are the same as in Fig. 11. b).

following equation must be used instead of where $\bar{Q}$ is the average quality factor in the equation (6).

$$
\begin{aligned}
& \ln A=\text { const }-\frac{\pi f}{\bar{Q}} \int \frac{d s}{V} \\
& \begin{array}{c}
\text { (ray path in } \\
\text { the outer core })
\end{array} \\
&=\text { const }-\frac{\pi t_{k}}{\bar{Q}} f
\end{aligned}
$$
outer core, and $t_{k}$ is the $S K S$ travel-time through the outer core.

Several cases are shown for the $\bar{Q}$ determination in Fig. 13, using the same data as (8) in the $\bar{\nu}$ determination. The $\bar{Q}$ values estimated from each pair are summarized in the 
Table 2. Results of $\bar{\nu}$ - and $\bar{Q}$. determination

\begin{tabular}{c|c|c|c|c}
\hline $\begin{array}{c}\text { Earthq. } \\
\text { No. }\end{array}$ & SKS & ScS & $\bar{\nu}\left(\times 10^{10}\right.$ c.g.s. & $\bar{Q}$ \\
\hline \multirow{5}{*}{1} & LPB (EW) & RIV (EW) & $3 \sim 5$ & $100 \sim 200$ \\
& QUI (EW) & RIV (EW) & 5 & $100 \sim 200$ \\
& ARE (EW) & RIV (EW) & $5 \sim 7$ & $100 \sim 200$ \\
& Average & & 5 & 150 \\
\cline { 2 - 5 } & NNA (EW) & TAU (EW) & $2 \sim 3$ & $200 \sim 300$ \\
\cline { 2 - 5 } & GIE (EW) & ADE (EW) & $2 \sim 3$ & $200 \sim 300$ \\
& COL (NS) & ADE (EW) & 10 & 60 \\
\hline \multirow{5}{*}{2} & LPB (EW) & RIV (EW) & $5 \sim 7$ & $100 \sim 200$ \\
& QUI (EW) & RIV (EW) & $3 \sim 5$ & $200 \sim 300$ \\
& ARE (EW) & RIV (EW) & $5 \sim 7$ & $100 \sim 200$ \\
& ANT (EW) & RIV (EW) & $3 \sim 5$ & $200 \sim 300$ \\
& Average & & 5 & $150 \sim 200$ \\
\hline & BOZ (EW) & *ADE (NS) & $1 \sim 2$ & $200 \sim 300$ \\
& ALQ (EW) & *ADE (NS) & $2 \sim 3$ & $300 \sim 500$ \\
\hline \multirow{5}{*}{3} & NAI (EW) & TRN (NS) & $2 \sim 4$ & $200 \sim 300$ \\
& *LON (NS) & ALQ (NS) & $6 \sim 8$ & 100 \\
& COR (EW) & ALQ (NS) & 10 & 50 \\
\hline & ESK (EW) & JCT (NS) & $1 \sim 2$ & \\
\hline
\end{tabular}

* indicates transverse component to the ray path.

right column of Table 2, and most of them are in the range from 100 to 300 .

We have investigated the attenuation in the outer core in two ways. Though we cannot conclude definitely in what way the seismic waves are attenuated through the outer core, it is most likely that the attenuation is like that in a viscous-liquid medium, where the viscosity coefficient $\nu$ is independent of frequency.

\section{$\S 5$. Discussion}

In the previous section we obtained the results that the outer core may be regarded as a viscous-liquid medium and the estimated values of the mean viscosity coefficient are in the range from $3 \times 10^{10}$ to $7 \times 10^{10}$ dyne $\cdot \mathrm{sec} /$ $\mathrm{cm}^{2}$. These value are consistent with the upper limit of $8.6 \times 10^{10} \mathrm{dyne} \cdot \mathrm{sec} / \mathrm{cm}^{2}$, which was found from multiply reflected $S c S$ phases by SATO and ESPINOSA (1968).
In this paper the seismograph responses are assumed to be the same for all the observing stations since the free periods of seismometer and galvanometer are approximately the same. To check this assumption, we calculated the seismograph response for several stations according to the method developed by ESPINOSA et al. (1965), and found the overall response of the system to be similar, to a first order approximation.

Concerning the correction on crustal structure necessary for the calculations of the transfer functions, there remains some ambiguity. The results of near profiles for some stations are directly available (for example, ADE, RIV, ALQ, and ARE), but for some other stations (for example, GIE and NAI) there are no profile data avaiable. For these cases the results of profiles having a similar geological structure were used. Since the spectral ratio not corrected for the crustal 
transfer functions is too irregular to determine the mean viscosity coefficient $\bar{\nu}$, the correction of the crustal transfer function will be necessary in a future investigation.

In the preceding sections we neglected the effect of lateral inhomogeneity in the upper mantle. It is shown here that the effect is negligible in the present $\nu$ determination.

Instead of equation (6) in section 2 , the following equation for the case of laterally inhomogeneous mantle is used;

$$
\text { In } A=\text { const }-f^{2} \nu J-\pi f \Delta q
$$

where $q=T / Q, T$ being the travel time through the upper mantle, and $\Delta q$ is the difference of $q$ between $S K S$ and $S c S$ recording stations.

Here we consider the lateral inhomogeneity effect in the velocity-and $Q$-structure for the the uppermost $200 \mathrm{~km}$ of the mantle. The travel-time from a point with $200 \mathrm{~km}$ in depth to the Moho discontinuity is $39 \mathrm{sec}$ for the $S K S$ phase at $\Delta s k s=70^{\circ}$, and $38 \mathrm{sec}$ for the $S K S$ phase at $\Delta s k s=130^{\circ}$. This means that the angle of incidence due to the difference in the velocity structure must be very small.

If the difference of $Q$ in the upper mantle, beneath the $S K S$ and the $S c S$ recording stations, is $\varepsilon$ then $\Delta q$ becomes $T / Q \varepsilon / Q$. Using values of $40 \mathrm{sec}, 150$ and 50 for $T, Q$ and $\varepsilon$, respectively, we get $\Delta q \doteqdot 0.09 \mathrm{sec}$. The third term on the right-hand side of equation (9) becomes $0.3 f$. Using values of $11 \times 10^{-10}$ and $5 \times 10^{10}$ for $J$ and $\bar{\nu}$, the second term becomes $55 f^{2}$. In the frequency range from $0.04 \mathrm{~Hz}$ to $0.15 \mathrm{~Hz}$ presented, the second term is greater than the third term by a factor of 1 . Therefore, the effect of lateral inhomogeneity in the upper mantle does not affect the $\bar{\nu}$ determination with the accuracy considered in the present study.

One of the severe constraints of the present investigation is the scantiness of the data which is available for this type of research. However, further investigation with more refined data will yield a detailed information on the attenuation model of the outer core.

\section{§6. Summary}

Attenuation of seismic waves in the outer core is investigated by utilising pairs of $S K S$ and $S c S$ phases which have common ray paths in the mantle, eliminating in this way the effects of crustal structure beneath the observing stations. Since the mechanism of attenuation in the outer core is not known at all, two mechanisms are considered. One is that the attenuation coefficient is proportional to the square of the frequency which. correspons to the attenuation in a viscousliquid medium. The other is that the attenuation coefficient is proportional to frequency, which corresponds to the attenuation in a. lossy solid.

Comparison of the theoretical curves under the above mentioned assumptions for a lossmechanism with the observed data suggests, but not definitely, that the attenuation in the outer core is interpreted well as a viscousliquid medium. In the frequency range from $0.04 \mathrm{~Hz}$ to $0.15 \mathrm{~Hz}$, the mean viscosity coefficient $\bar{\nu}$ is estimated to be $3 \times 10^{10}$ to $7 \times 10^{10}$ dyne. $\mathrm{sec} / \mathrm{cm}^{2}$.

\section{§7. Acknowledgements}

The authors are indebted to Dr. A. F. Espinosa who read the manuscript critically and offered valuable suggestions. They are grateful to Professor T. Asada for his encouragement during the course of the present study. All the members of Seismological Division of Geophysical Institute, Faculty of Science, University of Tokyo, contributed valuable help and advice.

Computations in this study were carried out by IBM-7090 through UNICOON in the International Business Machine Cooperation of Japan, to whom the author's sincere thanks. are also due.

\section{References}

Adams, R. D., and M. J. Randall, The fine structure of the Earth's core, Bull. Seism. Soc. Amer., 54, 1299-1313, 1964.

Anderson, D. L., and C. B. Archambeau, The anelasticity of the Earth, J. Geophys. Res., 69, 2071-2084, 1964.

Anderson, D. L, and R. L. Kovach, Attenuation in the mantle and rigidity of the core from 
multiply reflected core phases, Proc. Natl. Acad. Sci. U.S., 51, 168-172, 1964.

Anderson, D. L., A. Ben-Menahem, and C. B. Archambeau, Attenuation of seismic energy in the upper mantle, J. Geophys. Res., 70, 1441-1448, 1965.

Bolt, B. A., The velocity of seismic waves near the Earth's center, Bull. Seism. Soc. Amer., 54, 191-208, 1964.

Espinosa, A. F., G. H. Sutton, and H. J. Miller, A transient technique for seismograph calibra. tion-manual and standard set of theoretical responses, Geophys. Lab., Inst. of Science and Tech., Univ. of Michigan, 1965.

Gutenberg, B., Physics of the Earth's Interior, Academic Press, New York, 1959.

Hirasawa, T., and K. Takano, Differential attenuation of $P$ waves as derived from a Hindu-Kush earthquake, J. Phys. Earth, 14, 49-57, 1966.

Jeffreys, H., and K. E. Bullen, Seismological Tables, British Association for Advance for Science, Gray-Milne Trust, 1967.

Jeffreys, $H$. , The times of the core waves (second paper), M.N.R.A.S. Geophys. Suppl., 4, 594-615, 1939.

Kanamori, H., Spectrum of $P$ and $P c P$ in relation to the mantle-core boundary and attenuation in the mantle, J. Geophys. Res,, 72, 559-571, 1967a.

Kanamori, H., Spectrum of short-period core phases in relation to the attenuation in the mantle, J: Geophys. Res., 72, 2181-2186, 1967b.

Kanamori, H., Attenuation of $P$ waves in the upper and lower mantle, Bull. Earthq. Res. Inst., 45, 299-312, 1967c.

Kovach, R. L., and D. L. Anderson, Attenuation of shear waves in the upper and lower mantle, Bull. Seism. Soc. Amer., 54, 1855-1864, 1964.
Kurita, T., Attenuation of long-period $P$-waves and $Q$ in the mantle, J. Phys. Earth, 14, 1-14, 1966.

Kurita, T., Attenuation of short-period $P$-waves and $Q$ in the mantle, J. Phys. Earth, 16, 61-78, 1963.

Mikumo, T., and T. Kurita, $Q$ distribution for long-period $P$ waves in the mantle, $J$. Phys. Earth, 16, 11-29, 1968.

Sato, R., Amplitudes of $P c P$ and $P c S$ obtained from ray and wave theory solutions and amplitudes near shadow boundary, J. Phys. Earth, 17, 1-12, 1969.

Sato, R., and A. F. Espinosa, Dissipation in the Earth's mantle and rigidity and viscosity in the Earth's core determined from waves multiphy reflected from the mantle-core boundary, Bull. Seism. Soc. Amer. 57, 829-856, 1967.

Steinhart, J. S., and R. P. Meyer, Explosion studies of continental structure, Carnegie Inst. Publ., 1961.

Summer, R. D., Attenuation of earthquake generated $P$ waves along the Western Flank of the Andes, Bull. Seism. Soc. Amer., 57, 173-190, 1967.

Takano, K., Attenuation of short Period $S$ waves in the mantle (in Japanese), Zisin 2, 19, 246-254, 1966.

Talwani, M., G. H. Sutton, and J. L. Worzel, A crustal section across the Puerto Rico Trench, J. Geophys. Res., 64, 1545-1555, 1959.

Teng, T. L., Body wave and earthquake source studies, Chäpt. 7, Attenuation of body waves and $Q$ structure of the mantle, Thesis, Cal. Inst. Tech., 1966.

Teng, T. L., Attenuation of body waves and the $Q$ structure of the mantle, J. Geophys. Res., 73, 2195-2208, 1968. 